\title{
PREVENCIÓN, PROMOCIÓN DE LA SALUD Y LA BIOÉTICA PERSONALISTA EN LA DEFENSA Y PROMOCIÓN DE LA VIDA HUMANA
}

\section{Prevention, Health Promotion and Personal Bioethics in the defense and promotion of human life}

\author{
Carla Elisa Morán Huanay \\ Universidad Femenina Sagrado Corazón carlamoranh@unife.edu.pe \\ ID ORCID 0000-0002-3944-5782
}

\begin{abstract}
RESUMEN
El presente artículo pretende realizar una reflexión en torno al tema de la Prevención, Promoción de la salud y la Bioética personalista en la defensa y promoción de la vida humana. Hoy más que nunca se hace necesario sostener una mirada vigilante hacia la vida humana, que continuamente se encuentra expuesta a peligros que afectan la salud y bienestar de las personas, de allí la necesidad de una incisiva promoción de la salud, que beneficie a todos. La Bioética Personalista en este contexto, se propone a través de tres de sus principios como aquel modelo que defiende a la persona humana y su dignidad, en la promoción del cuidado de la salud y la vida de cada persona, como un compromiso tanto individual como colectivo.
\end{abstract}

\section{Palabras clave:}

Salud, prevención, promoción de la salud, educación para la salud, Bioética.

\begin{abstract}
This paper aims to reflect on the theme of Prevention, Health Promotion and Personal Bioethics in the defense and promotion of human life. Today more than ever it is necessary to maintain a vigilant look at human life, which is continually exposed to dangers that affect the health and well-being of people, hence the need for an incisive health promotion that benefits everyone. Personalistic Bioethics in this context is proposed through three of its principles as the model that defends the human person and its dignity, in the promotion of health care and the life of each person, as both an individual and a collective commitment.
\end{abstract}

\section{Keywords:}

Health, prevention, health promotion, health education, Bioethics. 


\section{INTRODUCCIÓN}

Hoy en día la salud viene concebida desde una interacción entre varios factores: personales, sociales, políticos, económicos, culturales y ambientales; es decir, desde una visión global. Los campos de la prevención y promoción de la salud han tenido a lo largo del tiempo un sustento teórico que ha logrado enriquecerse de diversos estudios e investigaciones, así como del aporte de diversos organismos a nivel mundial, incorporando cada vez más, nuevos elementos que permitan responder al ritmo acelerado del desarrollo a nivel mundial y a las necesidades reales de cada sociedad en continuo cambio.

Sin embargo, en la práctica muchas veces se evidencia por un lado la ausencia de una conciencia real de prevención, basada en la responsabilidad individual del ciudadano frente a la propia salud y la propia vida, pero también en la responsabilidad por parte de la sociedad y de las instituciones de gobierno, donde se denota una escasa atención en la creación de políticas públicas eficaces en el sector salud, que puedan responder a las necesidades efectivas de cada población, incluyendo el empleo de recursos y estrategias para la promoción de la salud, entre las que se encuentra la educación para la salud, que se configura como el mejor aliado, al potenciar los recursos internos de cada persona en el constate cuidado de la propia salud y de la propia vida.

Así como la educación para la salud se convierte en un fuerte aliado para la promoción de la salud, también la Bioética se propone como responsable frente al cuidado de la vida humana y por ende frente a la promoción de la salud, plasmando para ello algunos principios orientadores que permiten iluminar esta tarea que involucra a toda la sociedad. Es por ello que el presente artículo realiza primero una mirada a la evolución del concepto de salud propuesto por la OMS, para realizar luego un breve recorrido en los temas de prevención y promoción de la salud, la educación en la promoción de la salud, finalizando con el aporte que nos ofrece la Bioética Personalista en la reflexión del campo de la promoción de la salud y la vida humana.

\section{Una aproximación a la evolución en la definición de salud de la OMS.}

Se puede afirmar que la definición de «salud», ha sufrido continuos cambios en su interpretación a lo largo del tiempo, respondiendo a una determinada condición histórica y asumiendo hoy en día un enfoque mucho más holístico, integral y claro, que involucra aspectos relevantes en la vida de la persona. La Organización Mundial de la Salud (OMS) formuló por primera vez el concepto de salud en 1948, definiéndola como un "Estado de completo bienestar físico, mental y social, y no solamente la ausencia de afecciones o enfermedades" (OMS, 1948). Tal definición llegó a superar una visión reduccionista que entendía la salud solamente como «ausencia de enfermedad», es decir, desde un punto de vista puramente biológico; advirtiendo así un enfoque más amplio en el concepto de salud, que también incorporaba las dimensiones social y psicológica e introduciendo por primera vez elementos subjetivos. De allí que la percepción personal con respecto a la propia salud viene considera como un indicador del estado de salud, junto al entorno en que se encuentra. La salud viene entonces asociada no solo a la condición de ausencia de enfermedad, 
sino también a la búsqueda de un estado de bienestar psicofísico de la persona.

No obstante, esta definición haya contribuido a proporcionar una visión «positiva» de la salud respecto a precedentes concepciones reduccionistas, todavía se han podido encontrar limites asociados a ciertas ambigüedades. Entre las críticas a este concepto se encuentran: - la salud como algo estático (estado), en lugar de dinámico y variable; - la salud como una utopía (completo), en lugar de ser un fin; - no se considera la diversidad cultural al interpretar la salud y los problemas en este campo; - identificar la salud con «bienestar» no siempre es correcto, ya que el bienestar no necesariamente implica estar con «salud».

Seppilli (1966), ofreció algunos elementos que permitieron una lectura más dinámica en el concepto de salud, diferenciándola ampliamente de la definición de la OMS: "La salud es una condición de equilibrio armonioso, físico y psíquico, del individuo, integrado dinámicamente en su propio ambiente natural y social" (MOVE EUROPE). La idea de equilibrio viene aquí presentada como algo que involucra el bienestar psicofísico de la persona y que se busca constantemente en su integración con el entorno y en las condiciones en que se encuentra; de esta manera se va determinando el papel de cada individuo frente a la propia salud, añadiendo el componente social en el concepto de salud.

Una visión más amplia al concepto de salud se expresa por primera vez en la Carta de Ottawa, expresión de la $1^{\text {a }}$ Conferencia Internacional sobre Promoción de la Salud, a través de la cual, subrayando la importancia del rol de la población y de las organizaciones en la creación de oportunidades y comportamientos saludables, en 1986 se planearon estrategias dirigidas a este propósito. En esta Carta, la salud viene a concebirse desde una visión dinámica, como un medio dirigido al logro de objetivos, como un recurso que permite a las personas lograr una vida armoniosa ya sea en la esfera personal como social: "[...] La salud se percibe pues, no como el objetivo, sino como la fuente de riqueza de la vida cotidiana. Se trata por tanto de un concepto positivo que acentúa los recursos sociales y personales, así como las aptitudes físicas" (Organización Mundial de la Salud, 1986). La salud vendría a actuar así, como un medio que nos permita alcanzar niveles adecuados de vida.

La Carta, establece además que: "[...] para alcanzar un estado adecuado de bienestar físico, mental y social, un individuo o grupo debe ser capaz de identificar y realizar sus aspiraciones, de satisfacer sus necesidades y de cambiar o adaptarse al medio ambiente" (OMS, 1986). De allí que «ser capaz» implique asumir una responsabilidad que compromete no solo al individuo sino también a la sociedad y a sus diversas estructuras, que deberían ofrecer los recursos necesarios para satisfacer las necesidades y aspiraciones de la población.

La OMS (1998), en su glosario sobre la Promoción de la Salud, refiriéndose a la Carta de Ottawa, subraya la necesidad de una visión integral de la salud que implica que todos los sistemas y todas las estructuras, actúen en favor de la salud, del bienestar de las personas y la comunidad entera. Resaltando el vínculo existente entre salud y condiciones sociales y económicas, el entorno físico y los estilos de vida individuales. 
La Constitución de la OMS del 2006, también señala al respecto "El goce del grado máximo de salud que se pueda lograr es uno de los derechos fundamentales de todo ser humano, sin distinción de raza, religión, ideología política o condición económica o social. La salud de todos los pueblos es una condición fundamental para lograr la paz y la seguridad, y depende de la más amplia cooperación de las personas y de los Estados" (OMS, 2014). Esta puntualización refleja el grado de responsabilidad, no solo del individuo, sino también de las instituciones y gobiernos en la promoción de la salud, considerando la fuerte influencia de las variables culturales y sociales, que implica una necesaria y pronta implementación de políticas sociosanitarias diferenciadas en cada país.

En las últimas décadas, se ha asistido a un crecimiento significativo por el interés en temas de salud pública y al surgimiento de diversos enfoques del concepto de salud, así como de estilos de vida que promueven la salud y bienestar de la persona. El concepto de salud ha pasado así por diversas interpretaciones a lo largo del tiempo, que pueden ser identificadas en tres paradigmas o modelos:

\section{El primer paradigma organicista-} reduccionista, coloca en el centro el cuerpo humano y no considera las otras dimensiones de la persona, por lo que el estado de salud viene definido solamente con criterios de eficiencia física y de ausencia de enfermedad. El paradigma de la OMS, que interpreta la salud como un estado de completo bienestar físico, mental y social y no solo como la ausencia de enfermedades, mira a la persona desde una visión integral, sin embargo, corre el riesgo de ofrecer una lectura estática de la salud, además de presentar una visión utópica de salud. Por último, el paradigma del dinamismo interpreta la salud desde una visión holística del ser humano, como un elemento que pertenece tanto a la experiencia cotidiana como a la vivencia subjetiva. Una idea de salud como equilibrio dinámico en constante búsqueda, una conquista de la salud vinculada con los comportamientos o estilos de vida adoptados. Se tiene salud en la medida en que se vive de manera consciente, libre y responsable, valorando y utilizando todos los recursos que se poseen en cada situación (Di Pietro-Faggioni, 2014).

Este último paradigma, acoge al ser humano en su totalidad, enfatizando el papel de la responsabilidad individual. La salud viene vista desde una perspectiva integral de la persona que vive en constante tensión hacia un equilibrio dinámico y que lo empuja a actuar siempre de manera responsable en favor de la propia salud.

Además de la primera Conferencia Internacional sobre la Promoción de la Salud de Ottawa en 1986, que tuvo un papel importante para la comprensión de la promoción de la salud, proporcionando directrices para la acción en este campo, se han realizado otras conferencias internacionales sobre Promoción de la Salud, algunas de las cuales se mencionan brevemente por su relevancia en el significado de la salud.

En la Declaración de Alma Ata sobre atención primaria de salud (1978), de la Conferencia Internacional de Atención Primaria de Salud, se viene a reafirmar que la salud es un derecho fundamental y que el máximo nivel de salud alcanzable vendría a ser un resultado social 
extremadamente importante que se debe alcanzar a nivel mundial, cuya realización y éxito requieren de la contribución de diversos sectores: económico y social, así como sanitario.

La Declaración de Yakarta (1997), de la IV Conferencia Internacional sobre Promoción de la Salud, reflexiona sobre lo recogido en dicha conferencia acerca de la eficacia de la promoción de la salud, destacando algunas prioridades principales tales como: la promoción de la responsabilidad social en salud, el aumento de la inversión en salud, consolidación y expansión de recursos que promueven la salud, empoderamiento de las personas, entendido como el proceso a través del cual las personas adquieren un mayor control sobre las decisiones y acciones vinculadas con la propia salud.

La Carta de Bangkok (2005), de la VI Conferencia Internacional sobre la Promoción de la Salud, destacó en ese entonces algunos elementos nuevos y desafíos determinantes en el campo de la salud (desigualdades, cambios ambientales, sociales, económicos, demográficos) y nuevas oportunidades para mejorar la salud (tecnología, comunicación, intercambio de experiencias); además, se describen los cuatro compromisos importantes para eliminar las desigualdades en salud, a través de la promoción, bajo la responsabilidad de cada estado, sociedad e instituciones a nivel mundial.

Los documentos, fruto de estos encuentros, tuvieron grandes repercusiones a nivel organizativo en el sector salud, con una fuerte influencia en la creación y consolidación de estrategias de prevención y promoción de la salud y en el incremento de recursos adecuados al contexto social y cultural, exigiendo responsabilidad y apoyo, tanto a nivel individual como colectivo.

En este contexto se insertan la prevención y promoción de la salud, consideradas como un deber moral del individuo y de la sociedad, dirigida a promover estilos de vida saludables y evitar los comportamientos que pueden causar la aparición de patologías o la ruptura del equilibrio psicofísico.

\section{Una mirada a la prevención en salud.}

El término prevención que deriva del latín praevenere y significa "venir primero" o "a tiempo", contempla también el significado "anticipar", anticipar algo negativo actuando antes, evitar que algo se manifieste actuando con celeridad. La acción preventiva no debería ignorar estos significados, que a su vez poseen un alcance pedagógico. Ese "anticipar" algo negativo, es lo que se presenta como un elemento clave en la prevención, manifestando la exigencia de intervenir antes que se manifieste un fenómeno negativo, dirigiéndose hacia la búsqueda de los factores causantes a fin de contrarrestar los efectos. Se trata entonces de evitar cuanto sea posible situaciones peligrosas o que constituyan una amenaza para el equilibrio de la persona.

La prevención en el campo de la salud actúa mediante el uso de estrategias o intervenciones enfocadas a individuos o grupos de personas, en referencia a un problema específico. Estas pueden ser diferenciadas en intervenciones o niveles de prevención. Las intervenciones preventivas se pueden clasificar en: intervenciones universales (dirigidas a todas las personas), intervenciones 
selectivas (dirigidas a personas con altos factores de riesgo o vulnerabilidad), intervenciones indicadas (dirigidas a personas con síntomas y signos objetivos de malestar). La prevención se puede ejecutar también desde los tres niveles que son conocidos como los momentos en los que se efectúa la prevención: La prevención primaria, dirigida a toda la población y tiene como propósito, evitar o reducir la aparición de síntomas de malestar o enfermedad, promoviendo la salud. Está centrada en reducir los factores de riesgo y en el incremento de los factores de protección, por lo que las estrategias de prevención primaria se dirigen a fomentar cambios en los estilos de vida o comportamientos perjudiciales para la salud. Un ejemplo son los comportamientos de riesgo, en cuya prevención desempeñan un papel fundamental la educación y la intervención de la familia. La prevención secundaria, dirigida a un individuo o a una población específica considerada a riesgo, busca ralentizar o detener la evolución de los primeros síntomas de enfermedad o malestar. Este tipo de prevención actúa a través de la detección temprana de problemas de salud, facilitando así cambios de conducta que permitan el tratamiento de las enfermedades. También viene definida por algunos como una intervención temprana con respecto a la manifestación de algunos síntomas de malestar y de cualquier comportamiento de tipo negativo asociado al mismo. Finalmente,

la prevención terciaria, tiene como objetivo reducir las consecuencias negativas de la enfermedad. Se trata específicamente de actividades de rehabilitación, con el objetivo de reintegrar a la persona que ha experimentado consecuencias negativas, es decir, se trata de ayudar a la persona ya enferma reduciendo al máximo las consecuencias de la enfermedad. En este caso algunos autores hablan directamente de intervenciones terapéuticas. De este modo, la prevención puede darse antes de que surja el problema procurando condiciones favorables (prevención primaria); cuando el problema se manifiesta en una etapa temprana, evitando su progreso (prevención secundaria); cuando el problema ya se ha desarrollado, interviniendo con el objetivo de evitarlo en un futuro o mitigar las consecuencias (prevención terciaria)

Existe un vínculo estrecho entre Prevención y Promoción. Para muchos autores, de hecho, el término prevención, en particular «prevención primaria», coincide con el de «promoción de la salud», dada la similitud entre enfoques y estrategias utilizadas. Es así como la prevención primaria se manifiesta como una herramienta que permite abordar positivamente la salud. "El término prevención primaria, en la medida en que la intervención no apunta tanto a eliminar riesgos específicos, sino más bien a favorecer actitudes, hábitos y comportamientos positivos generales, también puede denominarse promoción de la salud" (Auxilia-Pontello, 2011 , p. 118$)$.

El enfoque preventivo parte del supuesto de que cada persona tiene la posibilidad de influir sobre la propia salud, ya que en ella se encuentran actuando múltiples factores de tipo comportamental, que implican autocontrol y responsabilidad directa de la persona frente a las propias acciones. Para lograr un trabajo eficaz de prevención, además de la colaboración del individuo, se hace necesaria la colaboración de la sociedad con sus estructuras; que, partiendo de objetivos comunes garanticen los recursos necesarios. 
También es importante considerar dentro de la acción preventiva la evaluación de la eficacia de las intervenciones realizadas, que contribuyen a mejorarlas o modificarlas, esto junto con el análisis crítico de los estudios e investigaciones realizados en este campo, que proporcionan una visión más clara de la realidad e identifican las necesidades urgentes, pues "[...] como en la clínica se habla de Medicina Basada en la Evidencia, también en el campo de la prevención se reconoce la importancia de evaluar la eficacia de las intervenciones a implementar" (Morandini, 2003, p.258).

La prevención se presenta, así como un recurso muy importante en el campo de la salud pública, logrando su propósito en la medida en que las instituciones se comprometan en activar servicios y promover intervenciones que estimulen y desarrollen una mayor responsabilidad y conciencia en la población sobre comportamientos que promueven la salud. Pero es necesario pasar de una visión que se enfoca en aquello que debe evitarse, a una visión que mejore los recursos positivos de la persona, buscando fortalecer el cuidado de la salud, mejorar la calidad de vida de las personas, creando las condiciones óptimas de bienestar.

\section{La promoción de la salud como responsabilidad personal y colectiva.}

La promoción de la salud se presenta según la OMS como el proceso que:

"permite que las personas tengan un mayor control de su propia salud. Abarca una amplia gama de intervenciones sociales y ambientales destinadas a beneficiar y proteger la salud y la calidad de vida individuales mediante la prevención y solución de las causas primordiales de los problemas de salud, y no centrándose únicamente en el tratamiento y la curación" (OMS, 2016).

Esta definición que parte de un concepto positivo de salud, se orienta a potenciar recursos y responsabilidades individuales y sociales. La promoción de la salud se conecta con estilos de vida y comportamientos que apuntan al bienestar de la persona. Se puede encontrar otra definición similar en la propuesta por O'Donnell (2009):

Health Promotion is the art and science of helping people discover the synergies between their core passions and optimal health, enhancing their motivation to strive for optimal health, and supporting them in changing their lifestyle to move toward a state of optimal health. Optimal health is a dynamic balance of physical, emotional, social, spiritual, and intellectual health. Lifestyle change can be facilitated through a combination of learning experiences that enhance awareness, increase motivation, and build skills and, most important, through the creation of opportunities that open access to environments that make positive health practices the easiest choice (p.4).

Esta definición de promoción de la salud nos ofrece además una consideración bastante sustanciosa en el tema, pues considera la promoción de la salud, no solo como una ciencia, sino también como un arte; motivado por el 
logro de una salud óptima y del cambio en los estilos de vida como medio para alcanzarla, sirviéndose para ello de la acción educativa y subrayando la visión holística y carácter dinámico de la salud que involucra los aspectos tanto físicos, como emocional, social, espiritual e intelectual.

Si bien es cierto la persona es sujeto y objeto de la promoción en sí (Di Pietro, 2013), es decir, el principal protagonista de la propia salud, esto no menoscaba la labor de la sociedad y las instituciones, que deben garantizar las condiciones mínimas para el logro de óptimos niveles de salud en toda la población. Como en el caso de la prevención, estamos hablando de una responsabilidad tanto del individuo como de la sociedad. La promoción de la salud debe acoger las necesidades de cada persona, eliminando diferencias y condiciones de desigualdad, para lo cual se deben considerar el sentido de justicia, la planificación apropiada y una necesaria acción educativa (Di Pietro, 2013). A nivel social, entonces se trata de remover aquello que dificulta la promoción de estilos de vida que promuevan una buena salud para toda la población, dirigiéndose hacia el logro de objetivos comunes. Esta idea viene también respaldada por la Organización Panamericana de la Salud, quien alienta la tarea por parte de los estados, de garantizar el acceso a la salud a toda la población, así como la creación y difusión de entornos que la promuevan.

De esta manera, se entiende la necesidad de que las personas asuman un rol protagónico y de responsabilidad frente a la propia salud, pero al mismo tiempo está la colaboración por parte de la sociedad que debe brindar el soporte y los recursos necesarios para el cumplimiento de este objetivo. Así, la promoción de la salud se hace cada vez más participativa y abierta al contexto y las necesidades de cada población.

La OPS también subraya la necesidad de un enfoque integral, un trabajo multidisciplinar en la Promoción de la salud, que involucre a diversos actores dentro del contexto social, para interactuar en favor de esta.

"[...] un enfoque de promoción de la salud implica un método multidisciplinario para promover la salud y prevenir enfermedades a través de un "sistema completo" en lugares o contextos sociales en los que las personas participan en actividades diarias, donde los factores ambientales, organizacionales y personales interactúan para afectar salud y el bienestar" (OPS, s.f.).

Respecto al rol de la sociedad para garantizar los recursos y acciones necesarias para promover la salud de sus ciudadanos, ya desde la Carta de Ottawa para la Promoción de la Salud, encontramos 5 líneas de acción claves para la promoción de la salud (OMS, 1986), que nos orientan hacia una reflexión en torno a la acción comunitaria en el esfuerzo por incrementar medidas de salud que beneficien y aumenten el bienestar de la población:

- Elaboración de políticas públicas saludables

- Creación y protección de entornos saludables

- Fortalecimiento de una acción comunitaria efectiva, que permita mejorar el nivel de salud. 
- Desarrollo de habilidades personales que garanticen el desarrollo individual y social y la toma de decisiones saludables.

- Reorientación de los servicios sanitarios, que se oriente más hacia la promoción de la salud.

Estos lineamientos, se propusieron como directrices de acción para los gobiernos en la implementación de medidas que hagan posible la construcción de estrategias y espacios que promuevan la salud en la población. A partir de este documento, los diversos estados del mundo se han involucrado progresivamente en la creación de herramientas apropiadas para este propósito, impulsados y ayudados también por los diversos documentos que en los años sucesivos han resultado de las diversas conferencias internacionales sobre Promoción de la Salud.

Ya más cerca, en el año 2016 en la $9^{\circ}$ Conferencia sobre Promoción de la Salud (OMS), se identificaron y señalaron tres pilares o componentes esenciales en la promoción de la salud y que fueron material de trabajo para dicha Conferencia: Buena gobernanza sanitaria, que implica la exigencia de dar un impulso a las políticas sanitarias en los organismos de gobierno, considerando la salud como un tema central dentro de las políticas públicas, así como también promover la creación y ejecución de diversas estrategias dirigidas a la práctica de hábitos de vida que incrementen el bienestar y la salud psicofísica de las personas. Ciudades saludables: con un rol central en la promoción de la salud, requieren del liderazgo y compromiso por parte de los gobiernos y autoridades para una organización eficiente en las estrategias y recursos destinados al logro de una ciudad con estándares de calidad en salud adecuados, en el compromiso de velar por el bienestar de la población. Las ciudades saludables contribuyen en la creación de países saludables y por tanto de un mundo más saludable. Educación sanitaria: como un medio importante en la adquisición de herramientas que hagan posibles decisiones en favor de la salud, a través de un aprendizaje continuo y la toma de conciencia frente a la propia salud, fortaleciendo la capacidad de realizar elecciones libres y responsables que favorezcan la salud y el bienestar de cada persona.

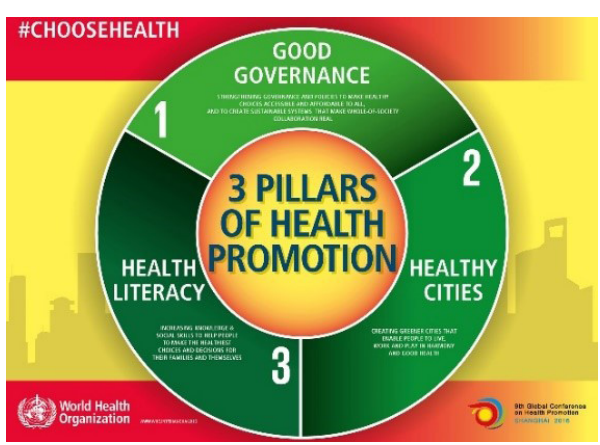

Figura 1. WHO (s.f.) 3 pillars of health promotion. Extraída de https://www.who. int/healthpromotion/conferences/9gchp/ resources/en/

Hoy en día, existe pues esta exigencia cada vez más grande de crear ambientes saludables, en grado de ofrecer posibilidades a todas las personas, garantizando la igualdad de oportunidades y recursos, a fin de que cada persona logre realizar plenamente su propio potencial. Si bien es cierto, se debe poner mucho énfasis en la creación de programas y estrategias empleadas en la promoción de la salud también se hace necesario que estas se adapten a las necesidades y posibilidades específicas de cada área geográfica y nación tomando en consideración los 
diversos sistemas culturales sociales y económicos de cada región.

El desarrollo de políticas y estrategias en favor de la salud, junto con entornos favorables, se deben constituir como parte de los objetivos principales del esfuerzo de cada sociedad que promueve a la persona y, en consecuencia, la salud de cada individuo. Por lo tanto, se trata de una promoción de esfuerzo individual y social, dirigida al logro de una mayor conciencia y cuidado de la salud, promoción que tiene como poderoso aliado a la educación sanitaria.

\section{La educación en la promoción de la salud}

Ahora bien, tanto el campo de la prevención como la promoción requieren de la acción educativa para transmitir sus contenidos y objetivos. De aquí la necesidad de insertar estos contenidos dentro de un contexto pedagógico, pues solamente la dimensión educativa de la prevención es capaz de proporcionar los recursos necesarios para promover la elección de comportamientos «positivos» y no solo para corregir comportamientos «negativos».

Es el ámbito educativo el que posee las condiciones necesarias para llevar a cabo esa tarea, ayudando en la adquisición consciente del propio actuar, en una fusión entre libertad y responsabilidad. Una educación para la salud tiene la tarea de valorar los recursos de la persona, recursos individuales y potencial que posee, para dirigirlos hacia una conciencia en el cuidado de la propia salud, que se traduzcan en elecciones libres y maduras. Por lo tanto, es necesario el esfuerzo y compromiso serio en el ámbito de la formación para la salud, "[...] una formación que no solo indique qué cosa hacer, sino que también ayude a cada uno a adquirir un habitus, una fuerza, una actitud permanente para la realización del propio bien" (Di Pietro-Faggioni, 2014, p.355). De aquí, la necesidad de implementar intervenciones destinadas a eliminar los factores de riesgo y promover en cambio los factores de protección, junto con la valorización de la responsabilidad social.

En una visión global de la salud, su promoción y protección se convierten, antes de un derecho, en un verdadero deber moral que se concretiza en la prevención o también en el cuidado por recuperar, en la medida de lo posible, la salud cuando la enfermedad ya la ha comprometido. Y es precisamente de la conciencia de esta dimensión moral que surgen la posibilidad y la necesidad de educar para la salud (Di Pietro,2008, p.61).

Se trata entonces de educar a la persona para que tome conciencia de su propio actuar, en la que deben fundarse la libertad y la responsabilidad. Responsabilidad por la propia vida y la vida de los demás, actuando siempre a favor de esta y de su promoción. La educación para la salud, aunque tiene sus inicios en la familia, también pasa por el contexto educativo formal desde la escuela primaria hasta la educación superior, ámbitos de formación que pueden considerarse lugares privilegiados para la promoción de la salud.

Educar para la salud se convierte en una contribución para el desarrollo y perfeccionamiento de la persona, sus habilidades y recursos, orientándolos positivamente. La intervención de la educación para la salud está dirigida a favorecer el desarrollo de cada persona en cuanto a conocimiento y habilidades personales, con el objetivo de aumentar las posibilidades 
de ejercer un mayor control sobre la propia salud, el propio comportamiento y el entorno en general. La educación para la salud también representa una oportunidad de aprendizaje, junto con la adquisición de un mayor conocimiento de las características de la población y el desarrollo de las habilidades personales que se dirigen hacia la salud personal y comunitaria. Así, esta educación no solo se limita a transmitir contenidos e información, sino también a estimular y desarrollar habilidades personales y de estima por la propia vida en las personas involucradas.

Es necesario a este punto recordar que la salud no es solamente el comportamiento libremente elegido por el individuo, sino también del entorno y escenario en el que se desarrolla tal comportamiento. Por tanto, la responsabilidad social e individual están como anteriormente mencionado estrechamente relacionadas. Este punto es importante en cuanto se puede caer en el error de culpar únicamente al individuo y no a la sociedad o a las estructuras que de ella dependen, prescindiendo de variables de tipo sociales, ambientales o económicas.

En la elaboración de intervenciones de prevención y promoción de la salud en las diferentes realidades y, en particular, en el campo educativo, también se hace necesario tomar en cuenta algunos elementos como: el estudio de la fase de desarrollo relacionada con la elaboración de los aspectos teóricos, el análisis de los contextos en que viven los sujetos, el uso de metodologías adecuadas, el compromiso de las instituciones involucradas y de la sociedad, en el estudio e investigación en este campo.

Las intervenciones de prevención y promoción que tienen un valor pedagógico, realizados a través de caminos de crecimiento y formación, deben partir de un análisis pertinente del grupo de personas con que se trabaja, considerando que en cada una de las etapas de vida de la persona hay tareas por realizar, pero también una serie de necesidades que deben satisfacerse para lograr un equilibrio adecuado en el crecimiento. Considerar el análisis desde las necesidades del grupo con el que se intenta trabajar es uno de los primeros pasos para la elaboración y ejecución de una estrategia eficaz de prevención y promoción de la salud. Por otra parte, la educación para la salud requiere de una continua evaluación de la eficacia de las intervenciones que se realizan en este campo; por lo tanto, no solo se trata de estructurar y activar programas de educación para la salud en diferentes contextos, sino que es necesario demostrar su efectividad y viabilidad, sometiéndolos a una revisión continua, para poder garantizar óptimos resultados.

\section{La Bioética Personalista en la defensa y promoción de la vida humana}

La Bioética personalista ofrece una importante contribución en el campo de la promoción de la salud y de la vida humana. Fundamentada en el Personalismo ontológicamente fundado coloca su acento en la centralidad de la persona.

La persona al centro de todas las acciones con una dignidad y un valor únicos. El personalismo reafirma la unidad de la persona como totalidad concreta (unidad de cuerpo y espíritu), integrada en sus dimensiones: física, psíquica y espiritual y poseedora de un valor intrínseco, por lo cual únicamente vale por aquello que es y no por las elecciones que realiza, presencia de ciertas características o funciones que presenta. Así la persona, en su unidad 
y en una constante tensión hacia la búsqueda de la perfección, tiene como una de sus principales tareas, desarrollar y madurar armoniosa y progresivamente cada una de las dimensiones de la propia vida.

Cuerpo y espíritu no solo pertenecen a la persona, sino que son la misma persona, una realidad que hacen posible la propia conciencia y el mundo exterior. Así la corporeidad nos ayuda a entender la esencia de la persona que no es solo cuerpo o solamente espíritu, sino que integra ambas realidades en una sola unidad. Tal como afirma Sgreccia (2009) "La corporeidad expresa la unidad corpóreo-espiritual de la persona. La persona es corpórea. Pero transciende el cuerpo, en el sentido de que tiene capacidad para ejecutar operaciones no exclusivamente corpóreas" (p.177).

La Bioética personalista realza así la dignidad de la persona humana, como poseedora de un valor absoluto, entendida como un fin en sí misma y protegiéndola de cualquier intento de ser instrumentalizada o ser vista como un medio. Una dignidad que es a la vez intransferible, propia de la unicidad y de la riqueza que representa cada persona, que no acepta diferencias ni discriminaciones. Dignidad que solamente puede ser reconocida, nunca atribuida, pues pertenece a la persona y no puede ser fruto de valoraciones subjetivas. Esta dignidad viene a ser realzada y reafirmada mediante actos buenos y propios de la persona en el desarrollo de cada una de sus dimensiones, incluyendo la física, en la cual la promoción de la salud constituye un elemento clave para el desarrollo de la persona.

El modelo personalista nos ofrece así cuatro principios que surgen del valor trascendente de la persona humana considerada en su totalidad: principio de defensa de la vida física, principio de totalidad o principio terapéutico, principio de libertad y responsabilidad, principio de sociabilidad y subsidiariedad. Pero son tres de sus principios en particular, los que nos ofrecen una valiosa contribución para la reflexión en el campo de la promoción de la salud.

\section{El Principio de defensa de la vida}

física, como su nombre lo indica nos compromete en la defensa de la vida y la promoción de la salud. Nos recuerda que la vida física o corporal representa el valor fundamental de la misma persona, la cual no agota toda la persona, sino que la trasciende pues es también espíritu.

Es a través de la vida física que la persona se hace visible, manifiesta su presencia (Di Pietro, 2012). Por medio del cuerpo la persona vive su propia historia, se expresa, se comunica, hace experiencia de la propia vida, hace realidad su proyecto de vida y su realización personal, se da en el encuentro con el otro y lo acoge; por ello el deber imperioso de protegerla, cuidarla, respetarla y promoverla en todo momento. Sgreccia (2009) nos lo recuerda: "[...] el respeto de la vida, así como su defensa y promoción, representan el primer imperativo ético del hombre para consigo mismo y para con los demás. Quizá sea necesario subrayar que no se trata solamente del respeto, sino también de la defensa activa y de la promoción"(p.219). Se trata pues, de una gran responsabilidad que compete en primer lugar a la misma persona, pero tam bién es colectiva, pues recae en toda la sociedad que debe velar y contribuir por la promoción de la vida de cada persona.

De este modo se pone de manifiesto la importancia de este principio en relación con la obligación moral de cuidar de la propia vida y promover la salud de 
cada ser humano. La salud como un bien indispensable derivado de la propia vida, que hace posible el bienestar de la persona, para lo cual es necesario tanto el compromiso por parte de la propia persona como de la sociedad. "Otra exigencia más: que la salud, valor subordinado y derivado de la vida, sea promovida para todos de manera proporcional a las necesidades de cada uno. No se trata de un derecho a la salud que ningún Estado pueda garantizar, sino del «derecho a los medios y a los cuidados indispensables» para la defensa y la promoción de la salud" (Sgreccia, 2009, p.221)

\section{El Principio de libertad-respon-} sabilidad, nos recuerda primero que antes que el derecho a la libertad está el derecho a la defensa de la vida, pues la vida es una condición necesaria para poder ejercer nuestra libertad. La condición de libertad o autonomía de la persona no puede prescindir de la responsabilidad, esencial en el actuar frente a la vida humana que estamos llamados a cuidar. Libertad que debe hacerse cargo no solamente de la propia vida sino también de la vida de los demás (Di Pietro, 2012).

Por lo tanto, el valor de la vida mencionado en el principio anterior coloca una exigencia a la libertad. Pues solamente un ejercicio responsable de nuestra propia libertad garantizará que las decisiones libres estén verdaderamente alineadas con la búsqueda del bien integral de cada una de las personas involucradas. Un claro ejemplo lo encontramos en la práctica clínica, donde este principio corrobora la obligación moral que tiene el paciente de colaborar con los cuidados ordinarios necesarios para salvaguardar tanto la propia vida como la ajena.
De esta manera, el principio de Libertad y responsabilidad nos compromete a velar por la propia salud y la de los demás, colocando todos los medios necesarios para cumplir con este objetivo, mediante una educación en la promoción de la salud y en la prevención de conductas o situaciones que coloquen en riesgo la salud y la vida de las personas. Labor que si bien es cierto en primer lugar es de la propia persona, también es competencia de cada estado o gobierno, que debe proporcionar los recursos y estrategias necesarias para el logro de estos objetivos.

\section{El Principio de sociabilidad y sub-} sidiariedad, también conocido para algunos autores como principio de solidaridad, mira la salud como un bien social. El principio de sociabilidad invita a mirar la propia vida y la vida de los demás como un bien común, y a la sociedad a promover el cuidado de la salud de todos, pues todos estamos involucrados con el cuidado de la vida y salud de los demás. Por ello compromete a la sociedad a "promover la vida y la salud de todos y cada uno, a fomentar el bien común promoviendo el bien de todos y cada uno" (Sgreccia, 2009, p.226), garantizando para ello todos los medios para poder acceder a los cuidados necesarios. "La sociedad es una verdadera sociedad cuando es solidaria" (García, 2013, p.68). Una sociedad solidaria, auténticamente humana y justa promueve el bien de cada persona comprometiéndose en su realización y al mismo tiempo en la realización del bien de los demás.

El principio de subsidiariedad nos recuerda que "todos deben recibir los medios necesarios para la promoción de su propia salud" (Di Pietro, 2012, p.59). Esto requiere el compromiso de la sociedad y de los gobiernos, quienes deben ga- 
rantizar estos medios para preservar $\mathrm{y}$ promover la salud de cada persona, evitando discriminaciones de cualquier tipo, gestionando con justicia y equidad los recursos sanitarios, priorizando la atención a los más vulnerables e interviniendo en aquellos casos de quienes no puedan satisfacer sus propias necesidades, también estimulando las iniciativas sociales que busquen el bien común en el cuidado de la salud; tal como menciona Sgreccia (2009): "[... proporcionar a todos en igual medida, los medios gratuitos de curación y asistencia sanitaria, promoviendo al mismo tiempo el respeto a la libertad de los ciudadanos y su participación activa" (p.227). Esto nos lleva también a ponderar la importancia por parte de los gobiernos de incidir cada vez más en la creación de políticas públicas que promuevan la salud para todos los ciudadanos, respondiendo así a las principales necesidades de la población, promoviendo a la vez la dignidad de la persona.

Estos tres principios del modelo de la Bioética Personalista constituyen un valioso aporte para al análisis y reflexión en el campo de la promoción de la salud y por tanto en la defensa y promoción de la vida humana, esto desde un enfoque que busca el desarrollo integral de la persona, realzando su dignidad y buscando su bien en todo momento, comprometiendo para ello no solamente a la misma persona sino también a toda la sociedad que se beneficia del bien de cada uno de sus miembros.

\section{CONCLUSIONES}

La definición de salud propuesta por la OMS en 1948, que nos presenta un enfoque positivo de salud, si bien es cierto ha presentado ciertas limitaciones que han sido objeto de muchas críticas; también a lo largo de los años posteriores a su aparición, se ha visto enriquecido gracias a los aportes de diversos estudiosos, diversas investigaciones en el campo de la salud, así como de las diversas conferencias internacionales sobre promoción de la salud, organizadas por la misma OMS, que nos han dejado una serie de documentos y directrices con grandes aportes a nivel organizativo en el sector salud, así como en la creación y consolidación de estrategias de prevención y promoción de la salud, con la esperanza que sean actuados a nivel mundial.

La prevención en salud, muy asociado a la promoción de la salud, constituye un recurso muy valioso al actuar eficazmente en cada uno de los niveles de prevención, pero sobre todo por el enfoque mediante el cual, a través de una prevención primaria, se busca reducir la aparición de factores de riesgo, incrementando mediante diversas estrategias los factores de protección con el fin de asegurar la salud y bienestar de la persona.

La promoción de la salud, instrumento clave en la promoción de la persona humana, exige un compromiso tanto individual como colectivo, capaz de eliminar brechas de desigualdad, integrando a todas las personas en esta importante tarea de promoción y posibilitando el acceso a los recursos necesarios para el logro de los objetivos. La Educación para la salud en este contexto, se constituye en un fuerte aliado a través de la implementación de programas y estrategias eficaces, acordes a cada población, contribuyendo así en el desarrollo de la persona y brindándole las herramientas necesarias ejercer un mayor control y cuidado sobre la propia salud.

La Bioética personalista, a través de tres de sus principios, nos propone un valioso aporte en la defensa y promo- 
ción de la vida humana. En el campo de la promoción de la salud, desde un enfoque centrado en la persona y la defensa de su dignidad en todo momento, enfatiza la responsabilidad y el compromiso no solamente individual sino también por parte de la sociedad, en el cuidado y en una constante promoción de la salud que beneficie a todas las personas.

\section{REFERENCIAS BIBLIOGRÁFICAS}

Auxilia, F y Pontello, M. (2011). Igiene e Sanità Pubblica. I fondamenti della prevenzione. Padova: Piccin Nuova Libraria.

Di Pietro, M.L. y Faggioni, M.P. (2014). Bioetica e infanzia: dalla teoria alla prassi. Bologna: EDB.

Di Pietro, M.L. (2013). Etica in Sanità Pubblica. Igiene, Medicina preventiva, Sanità pubblica, 764 780.

Di Pietro, M.L. (2008). Bioetica e famiglia. Città del Vaticano: Lateran University Press.

García, J.J (2013). Bioética Personalista y Bioética Principialista. Perspectivas. Cuadernos de Bioética. XXIV (1) 67.

Morandini, S. (2003). Etica e Stili di vita. Padova: Gregoriana Libreria Editrice.

Move Europe. Campagna Europea per la promozione della salute nei luoghi di lavoro. Cosa è la Promozione della salute. Recuperado de http:// www.move-europe.it/Salute.htm

O'Donnell, M.P. (2009). Definition of Health Promotion 2.0:
Embracing Passion, Enhancing Motivation, Recognizing Dynamic Balance, and Creating Opportunities. American Journal of Health Promotion. 24(1), iv. Recuperado de https://journals.sagepub.com/doi/ abs/10.4278/ajhp.24.1.iv

Pan American Health Organization (PAHO) (2013). Carta de Ottawa para la Promoción de la Salud 1986. Recuperado de https://www.paho.org/ hq/dmdocuments/2013/Carta-deottawa-para-la-apromocion-dela-salud-1986-SP.pdf

Pan American Health Organization (PAHO). Promoción de la salud. Recuperado de https://www.paho.org/ hq/index.php?option $=$ com_topicsEview = articleEid = 144E-lan$\mathrm{g}=\mathrm{es}$

Sgreccia, E. (2009). Manual de Bioética I. Fundamentos y ética biomédica. Madrid: Biblioteca de Autores Cristianos.

World Health Organization. ¿Cómo define la OMS la salud? Recuperado de https://www.who.int/healthpromotion/conferences/9gchp/shanghai-conference-report/en/

World Health Organization (1998). Promoción de la Salud: glosario. Recuperado de https:// apps.who.int/iris/bitstream/ handle/10665/67246/WHO_HPR_ HEP_98.1_spa.pdf?sequence $=1$

World Health Organization (2006). Constitución de la Organización Mundial de la salud. Recuperado de https://www.who.int/governance/eb/ who_constitution_sp.pdf 
World Health Organization. Promoting health in the SDGs. Report on the 9th Global Conference for Health Promotion: All for Health, Health for All, 21-24 November 2016. Recuperado de https://www.who.int/healthpromotion/conferences/9gchp/shanghai-conference-report/en/

World Health Organization. ¿Qué es la promoción de la salud? Recuperado de https://www.who.int/healthpromotion/conferences/9gchp/shanghai-conference-report/en/

Fecha de recepción: 18-04-2020

Fecha de aceptación: 10-05-2020 